\title{
Skull stripping of MRI brain images using mathematical morphology
}

\begin{abstract}
Skull stripping is a major phase in MRI brain imaging applications and it refers to the removal of its non-cerebral tissues. The main problem in skull-stripping is the segmentation of the non-cerebral and the intracranial tissues due to their homogeneity intensities. As morphology requires prior binarization of the image, this paper proposed mathematical morphology segmentation using double and Otsuôs thresholding. The purpose is to identify robust threshold values to remove the non-cerebral tissue from MRI brain images. Ninety collected samples of T1-weighted, T2-weighted and FLAIR MRI brain images are used in the experiments. The results showed promising use of double threholding as a robust threshold value in handling intensity inhomogeneities compared to Otsuôs thresholding.
\end{abstract}

Keyword: Skull stripping; Mathematical morphology; MRI; Thresholding 